\title{
Sleep in elderly with due emphasis on dementia
}

\begin{abstract}
Sleep is an extremely important aspect for maintaining health. Ageing is accompanied by various sleep complaints and sleep disorders. Similarly dementia is associated with different types of sleep and circadian disturbances, depending on the etiology of dementia. Medications for underlying dementia as well as medications for other comorbid conditions may also contribute to sleep disturbance.
\end{abstract}

Sleep disturbance in elderly age group should thus be considered as a multifactorial geriatric health condition. This requires a comprehensive treatment approach. This paper will review both normal and abnormal sleep in the elderly, with emphasis on sleep disorders in dementia.

Keywords: sleep disorders, geriatric health condition, dementia, insomnia, sleep architecture
Volume 2 Issue 2 - 2018

\author{
SC Tiwari, 'Akanksha Sonal ${ }^{2}$ \\ 'Professor and Head, Dept of Geriatric Mental Health, India \\ ${ }^{2}$ Senior Resident, Dept of Geriatric Mental Health, India
}

Correspondence: SC Tiwari, Professor and Head, Dept of Geriatric Mental Health, KGMU, India,

Emailsarvada1953@gmail.com

Received: October 31, 2017 | Published: March 22, 2018
Abbreviations: RLS, restless leg syndrome; OSA, obstructive sleep apnea; AD, alzheimer's disease; DLB, dementia with lewy bodies; PD, parkinson's disease; LBD, lewy body disease; MAO-B, monoamine oxidase-B

\section{Introduction}

Sleep is a crucial element for health and wellbeing across all age groups. There are usual age-related, normal changes that occur in sleep architecture and sleep patterns. However, ageing is also accompanied by various sleep complaints and sleep disorders. This paper will review the available literature on normal and abnormal sleep in the elderly, with a special focus on sleep disorders in dementia.

Sleep complaints are common in individuals above 65 years. ${ }^{1}$ The population of individuals above 65 years is steadily increasing and so will the prevalence of sleep disturbances. With ageing, there are changes in sleep architecture that are not in themselves pathologic, but which can be viewed as making older adults more vulnerable to sleep disturbances. ${ }^{2}$ Consequences of ageing, in the form of medical and psychiatric co-morbidity, medication and substance use, psychosocial factors and primary sleep disorders put older adults at risk for sleep disturbance. Thus, sleep disturbance in this age group should be considered as a multifactorial geriatric health condition requiring a comprehensive treatment approach.

\section{Discussion}

\section{Age related changes to sleep wake physiology in healthy ageing}

Physicians treating sleep disorders in elderly are commonly enquired as to 'how much sleep is enough'. The National Sleep Foundation recommends 7 to 8 hours of sleep for adults aged 65 and older. ${ }^{3}$ Evidence that older adults sleeping 6 to 9 hours have better mental and physical health, quality of life and cognition compared to older adults with shorter or longer sleep durations, supports this recommendation. ${ }^{4,5}$ The ability to get the required sleep may be decreased owing to normal changes in sleep architecture with ageing. ${ }^{6}$ Total sleep time declines by an average of 10 minutes per decade. ${ }^{5}$ Age-related changes in sleep physiology have been depicted in Table 1. Ageing is associated with a phase advance in circadian rhythm, resulting in an earlier onset of sleepiness in the evening and earlier morning awakening. ${ }^{7}$ Daytime wakefulness is also affected by this phase advance, with older adults being more alert in the morning and more somnolent in the evening.

Table I Age-related change in sleep architecture as studied by a polysomnography. ${ }^{8}$

\begin{tabular}{lll}
\hline & Decreased & Increased \\
\hline & Total sleep time & $\begin{array}{l}\text { Total awake after sleep } \\
\text { onset }\end{array}$ \\
Sleep & Sleep efficiency & $\begin{array}{l}\text { Number of arousals from } \\
\text { sleep }\end{array}$ \\
parameter & Slow wave sleep & Sleep latency \\
& Rapid eye movement sleep & \\
\hline
\end{tabular}

\section{Sleep complaints in older adults}

Approximately $40 \%$ to $70 \%$ of older adults have chronic sleep problems, and up to $50 \%$ of these go undiagnosed. ${ }^{8}$ Common sleep complaints include insomnia and drowsiness. Symptoms of insomnia include difficulty in initiating or maintaining sleep (includes early morning awakening). ${ }^{9}$ Drowsiness involves the propensity for sleep and is often established by napping behavior. ${ }^{10}$ The predominating sleep complaint depends on the cause of the sleep disturbance. Symptoms of insomnia are common in people with co-morbid medical or psychiatric illness, in those using activating substances or medicines, or in those with restless leg syndrome (RLS). Daytime drowsiness can result from chronic medical illness, sedating 
medications, or obstructive sleep apnea (OSA). Insomnia complaints are associated with harmful outcomes, which include cognitive decline, depression and disability in basic activities of daily living, poorer quality of life and a greater risk of institutionalization. ${ }^{2,11}$ It is also associated with impaired physical function and an increased risk for falls. ${ }^{11,12}$ Daytime drowsiness is associated with harmful outcomes, including cardiovascular disease, falls, and death. ${ }^{2}$

\section{Sleep complaints and dementia}

Dementia is associated with sleep and circadian disturbances, poorer than the expected sleep quality corrected for age. ${ }^{6}$ This negatively impacts patient quality of life and increases caregiver burden. This disturbed sleep and circadian functions in dementia can be attributed to neuro degeneration of brain regions involved in these functions, such as the suprachiasmatic nucleus. ${ }^{13}$ The delay in circadian phase contributes to sun downing, which leads to confusion and agitation in the evening, as well as to difficulty sleeping at night. Due to wandering and subsequent risk of injury, nighttime insomnia directly increases morbidity and mortality, and therefore is a common reason for institutionalization. During daytime, excessive sleepiness contributes to poor cognition, unintentional naps thereby impacting driving safety, and decreased ability to engage in social functions and therapies. There is thus need for identifying effective treatments, with the aim of improving patient quality of life, reducing caregiver burden, postponing institutionalization, and possibly slowing cognitive decline.

Various etiologies of dementia are associated with different types of sleep and circadian disturbances. In Alzheimer's disease (AD), $44 \%$ of patients are affected with a sleep disorder. ${ }^{14}$ The prevalence and severity of sleep disorders are directly proportional to the severity of dementia. Sleep disturbances occur very early in $\mathrm{AD}$. Even the preclinical stage of $\mathrm{AD}$ is associated with poor sleep quality and shorter sleep duration. ${ }^{15}$ OSA, a primary sleep disorder, is particularly common in AD as well as in vascular dementia. Dementia with Lewy bodies (DLB) and Parkinson's disease (PD) with dementia are pathologically similar and can be grouped together as Lewy Body Disease (LBD). LBD has the highest prevalence of sleep and circadian disturbances of any dementia, affecting approximately $90 \%$ of patients. ${ }^{16}$ Insomnia is the most common sleep disturbance in LBD. Another primary sleep disorder associated with PD is RLS, with a prevalence of approximately $20 \% .{ }^{17}$ In addition to these sleep and circadian disturbances associated with various dementias, there are additional factors that may worsen symptoms or complicate treatment. Co-morbidities that cause pain or discomfort, or psychiatric conditions such as depression may worsen nighttime insomnia. ${ }^{16}$ Medications for underlying dementia as well as medications for co-morbid conditions (e.g. $\beta 2$ agonist inhalers for pulmonary disease, anti-hypertensive medications) may contribute to sleep disturbance. Sleep hygiene, which includes the regularity and timing of sleep, napping, bedtime ritual, daytime activity, light and nocturnal noise, may be poor in dementia and this therefore may exacerbate sleep-wake problems. ${ }^{14} \mathrm{~A}$ comprehensive approach is thus necessary for diagnosis and treatment of sleep disorders in dementia.

Assessment of sleep and circadian disturbances in dementia includes a complete history. Since demented people may not recall symptoms accurately, history from caregivers is essential. The clinical history should assess for symptoms of primary sleep disorders, such as snoring, hypersomnia, witnessed apneas, parasomnias, restless legs, and leg movements during sleep. The timing and regularity of nighttime sleep and daytime naps (intentional and inadvertent) are important to know. In addition to these clinical features, individuals with dementia should also be enquired about sun downing, hallucinations, sleep attacks, injurious parasomnias, and night-time wandering.

The treatment approach ${ }^{18}$ to sleep problems in dementia is similar to that in the general population, with additional attention being paid to avoid exacerbating cognitive dysfunction, reducing injury risk, and reducing caregiver burden. Firstly, any underlying primary sleep disorders should be assessed for and treated. Secondly, any co-morbid mood and anxiety disorders should be treated. Thirdly, pain, nocturia, or other co-morbid conditions that interfere with sleep should be treated to the best extent possible. Medicines that affect sleep (including those for underlying dementia) should be adjusted to optimize sleep-wake cycle. For example, acetylcholinesterase inhibitors (e.g. Donepezil and Rivastigmine) and MAO-B inhibitors (e.g. Selegiline) may cause insomnia, and hence dosing of these should be moved earlier in the daytime. Also, dopaminergic medications for Parkinsonism should be adjusted to minimize nighttime motor symptoms that may awaken the patient, as well as minimize sedating effects during the daytime (especially dopamine agonists). Lastly, if sleep-wake problems persist, non-pharmacological treatments are preferred over pharmacological treatments. Pharmacological treatment has risk of sedation, cognitive symptoms, falls, injuries, and medication interactions. In recalcitrant cases, pharmacological treatments can also be added cautiously. There is some beneficial role of sleep hygiene education, physical and social activity, bright light therapy, and melatonin supplementation, without significant side effects. There are scant data to support the use of hypnotic medications for sleep disturbances in dementia, with only one small study showing a benefit of low-dose Trazodone. ${ }^{19}$ Response to treatment should be serially followed with objective measurements such as actigraphy and subjective measurements of patient and caregiver symptoms.

\section{Conclusion}

Sleep disturbance is common in older adults because ageing is associated with an increasing prevalence of multi morbidity, polypharmacy, psychosocial factors affecting sleep, and certain primary sleep disorders. Evaluation and management of sleep disturbance in older adults is an area of important focus. Because many older adults will have several factors from different domains affecting their sleep, these complaints are best approached as a multifactorial geriatric health condition, necessitating a multifaceted treatment approach.

Sleep and circadian disturbances are common in all types of dementia, and can manifest in array of symptoms. The clinical approach to sleep and circadian disorders in dementia begins with assessing for and treating primary sleep disorders, managing comorbid conditions and medications that may be negatively affecting sleep, then behavioral interventions, and then pharmacological treatments in that order. Due to the risk of sedation, falls, and worse cognitive function from hypnotic medications, they should be used very cautiously, with frequent serial assessments for safety and objective measurements of drug efficacy. Well designed studies are needed to study sleep and circadian disturbances in dementia population, with emphasis on treatment strategy. 


\section{Acknowledgements}

None.

\section{Conflict of interests}

The authors declared that there are no conflicts of interest.

\section{References}

1. Cooke JR, Ancoli-Israel S. Normal and Abnormal Sleep in the Elderly. In: PJ Vinken, GW Bruyn, editors. Handbook of Clinical Neurology. 2011; 98:653-665.

2. Vaz Fragoso CA, Gill TM. Sleep complaints in community-living older persons: a multifactorial geriatric syndrome. J Am Geriatr Soc. 2007;55(11):1853-1866.

3. Hirshkowitz M, Whiton K, Albert SM, et al. National Sleep Foundation's sleep time duration recommendations: methodology and results summary. Sleep Health. 2015;1(1):40-43.

4. Ancoli-Israel Sonia. Sleep and its disorders in aging populations. Sleep Medicine. 10(Suppl 1):S7-S11.

5. Ohayon MM, Carskadon MA, Guilleminault C, et al. Meta-analysis of quantitative sleep parameters from childhood to old age in healthy individuals: developing normative sleep values across the human lifespan. Sleep. 2004;27(7):1255-1273.

6. Avidan AY. Normal sleep in humans. In: Kryger MH, Avidan AY, Berry $\mathrm{RB}$, editors. Atlas of clinical sleep medicine. $2^{\text {nd }}$ ed. Philadelphia: Saunders; 2014. p. 65-97.

7. Monk TH. Aging human circadian rhythms: conventional wisdom may not always be right. J Biol Rhythms. 2005;20(4):366-374.

8. Edwards BA, O’Driscoll DM, Ali A, et al. Aging and Sleep: Physiology and Pathophysiology. Semin Respir Crit Care Med. 31(5):618-633.

9. Ohayon MM. Epidemiology of insomnia: what we know and what we still need to learn. Sleep Med Rev. 2002;6(2):97-111.

10. Johns MW. Sleepiness in different situations measured by the Epworth Sleepiness Scale. Sleep. 1994;17(8):703-710.

11. Bloom HG, Ahmed I, Alessi CA, et al. Evidencebased recommendations for the assessment and management of sleep disorders in older persons. $J$ Am Geriatr Soc. 2009;57(5):761-789.

12. Bliwise DL. Normal aging. In: Kryger MH, Roth T, Dement WC, editors. Principles and practice of sleep medicine. 6th ed. Philadelphia: Elsevier; 2016. p. 25-38.

13. Swaab DF, Fliers E, Partiman TS. The suprachiasmatic nucleus of the human brain in relation to sex, age and senile dementia. Brain Res. 1985;342(1):37-44.

14. Vitiello MV, Borson S. Sleep disturbances in patients with Alzheimer's disease: epidemiology, pathophysiology and treatment. CNS Drugs. 2001;15(10):777-796.

15. Ju YE, McLeland JS, Toedebusch CD, et al. Sleep quality and preclinical Alzheimer disease. JAMA Neurol. 2013;70(5):587-593.

16. Guarnieri B, Adorni F, Musicco M, et al. Prevalence of sleep disturbances in mild cognitive impairment and dementing disorders: a multicenter Italian clinical cross-sectional study on 431 patients. Dement Geriatr Cogn Disord. 2012;33(1):50-58.

17. Rijsman RM, Schoolderman LF, Rundervoort RS, et al. Restless legs syndrome in Parkinson's disease. Parkinsonism Relat Disord. 2014;20(1):S5-S9.

18. Sharon Ooms, Yo-El Ju. Treatment of sleep disorders in dementia. Curr Treat Options Neurol. 2016;18(9):40.

19. Camargos EF, Louzada LL, Quintas JL, et al. Trazodone improves sleep parameters in Alzheimer disease patients: a randomized, double-blind, and placebo-controlled study. Am J Geriatr Psychiatry. 2014;22(12):1565-1574. 